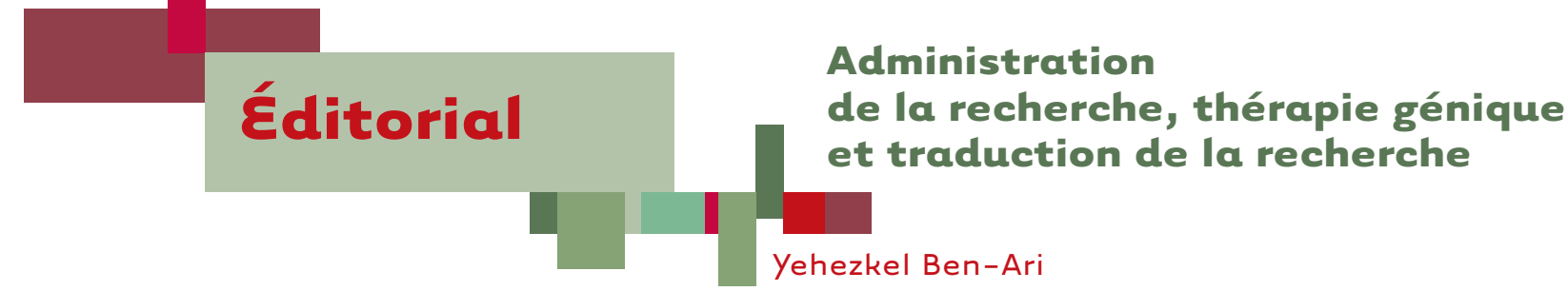

> Nous vivons une époque formidable !

La généralisation de la quantification à tout prix des qualités d'une recherche et de l'évaluation des chercheurs (une note et un tableau pour simplifier le travail administratif), des brevets comme haute distinction d'une rentabilité qui va immanquablement résoudre le problème du chômage, une évaluation basée sur les scoop publiés par des revues à «fort impact» dont la durée de vie est souvent celle d'un soupir, et une mise sous tutelle de la recherche à l'ancienne dans laquelle on pouvait encore vouloir passionnément comprendre un mécanisme, s'extasier devant la sagesse de mère nature et prendre des risques pourtant seules avenues permettant justement de découvrir les Graal qui vont permettre d'améliorer la vie de nos compatriotes et guérir les maladies qui résistent souvent aux traitements que nous proposons. Dommage, car au passage on aura perdu aussi bien notre spécificité que l'attrait de la nouvelle génération qui déserte sans états d'âme nos facultés des sciences au profit d'écoles de commerce.

Quand on lit les appels d'offres européens avec des déclarations tonitruantes du style: «nous voulons des projets à risque », mais qu'il est en même temps impératif de dire exactement ce que nous allons découvrir dans 14 à 18 mois... et confirmer ensuite que nous avons bien découvert ce que nous avions prévu, cela n'est plus de la recherche, c'est du retour sur investissement, des prises de risque sans risque, bref une vision économiste (quand celle-ci est raisonnable). Le pire c'est que même les fondations caritatives s'y mettent et rendent l'administration impossible et l'utilisation des fonds kafkaïenne. Les sommes promises doivent être d'emblée utilisées comme prévu, les overhead qui auraient pu donner un peu de souplesse ne servent finalement pas à grand-chose car encadrés à la virgule près. On ne peut plus payer un étudiant en thèse, changer d'avis et acheter un appareil imprévu, sauf à remplir $x$ formulaires et accroître encore le pourcentage des ressources durement acquises et destinées à recruter encore plus d'administratifs. La recherche est au service de l'administration. On pourrait penser que tout cela n'est qu'administratif, sauf que cela se conjugue avec un assèchement des moyens et, donc, une plus grande dépendance dans des ressources externes, notamment caritatives, et cela a forcément un coût. Ainsi, bon nombre d'équipes sont bien obligées de changer de sujet et d'aller là où il y a des moyens, ce qui se traduit par une perte de «biodiversité », une plus grande concentration au profit de domaines à la mode et la boucle est bouclée : plus de lisibilité, plus d'attractivité, donc plus de moyens, etc.

Prenons un exemple: la thérapie génique des maladies neurologiques. On assiste à une véritable «génétomanie » due à l'extraordinaire essor des approches génétiques qui a révolutionné nos outils et nous permet de faire des choses encore impensables il y a peu. L'envers de la médaille est que la génétomanie permet des messages faciles, se «vend » bien et colle bien avec des promesses tous azimuts - demain on guérit tout - y compris dans des domaines comme les maladies neurologiques où la thérapie génique reste improbable: les maladies majoritaires sont à faible contribution génétique ou mettent en jeu des complexes multigéniques et, surtout, nous devons prendre en compte l'extraordinaire plasticité des réseaux de neurones qui s'adaptent et réagissent à la mutation. Ainsi, bon nombre de mutations commencent à faire des dégâts in utero, générant des réseaux aberrants qui vont empêcher la construction d'un réseau cortical fonctionnel [1]. Si l'on veut précéder ces réarrangements, il faut intervenir très tôt. Dans un modèle animal utilisant une stratégie d'interférence ARN in utero de la double cortine - protéine impliquée dans la migration et dont la mutation induit la formation d'un double cortex avec retard mental et épilepsies -, on observe un double cortex chez les rongeurs. Si l'on essaie ensuite de corriger cela en introduisant les bons gènes par thérapie génique, on s'aperçoit que la réparation par transfection du bon gène n'est possible que pendant la première semaine de vie post-natale, donc in utero chez I'homme; après, c'est trop tard [1]. On comprend les limites de l'approche. Curieusement, l'invalidation in utero de protéines impliquées dans d'autres maladies «tardives » comme la maladie d'Alzheimer induit aussi des désordres migratoires. On comprend dès lors la difficulté de thérapies correctives de type génétique.

Les sommes énormes investies dans le tout génétique par I'AFM sont discutables à cette aune et il est regrettable que les fondations les plus généralistes qui aident la recherche en fonction de la qualité du projet sans ornière thématique comme la FRM soient du coup moins dotées. Sur un plan plus conceptuel, tout se passe comme si on espérait encore guérir des maladies sans en comprendre la genèse et les mécanismes ou pour utiliser des termes à la mode, «traduire» les 
découvertes en médicaments. Pour traduire une langue, il faut peut-être un peu la comprendre d'abord. Manquent cruellement à l'appel dans ce but des experts en physiologie, modèles animaux, biochimie, anatomopathologie etc., sciences asséchées par les concentrations financières, administratives, et la recherche du court terme. Combien de laboratoires étudient encore les malformations liées aux anoxies fœtales, aux accidents cérébro-vasculaires, aux épilepsies et traumas crâniens, le vieillissement cérébral des neurones, des vaisseaux, etc., pourtant principaux fournisseurs de maladies et de séquelles neurologiques.

Il convient, par conséquent, de respecter «les fondamentaux de la recherche », à savoir sa biodiversité intrinsèque, le soutien du projet en fonction de son intelligence et de sa qualité, sans tenir compte des applications promises de façon artificielle. Le problème du passage des découvertes aux applications est plus dû au cloisonnement de la recherche et à une incapacité certaine des décideurs privés comme publics à voir puis exploiter les découvertes faites. Pasteur avait bien résumé le débat en disant «il n'y a pas de recherche appliquée, il y a des applications de la recherche fondamentale ».

Je prendrai deux exemples pour terminer.

Après des années de discussions, la haute autorité européenne des médicaments a - enfin - décidé que les médicaments autorisés pour utilisation chez des enfants devront avoir été testés sur de jeunes animaux. Jusqu'à maintenant, pour donner un médicament à un nourrisson, il suffisait de prendre un médicament utilisé chez l'adulte et de faire un ratio en fonction du poids! Cela est assez étonnant, car quiconque a travaillé un peu sur le cerveau immature sait que tout ou presque est différent du cerveau adulte, y compris les propriétés pharmacologiques des courants ioniques qui peuvent parfois se traduire par des effets opposés sur le cerveau de la mère et de son embryon [3]. Problème de communication ou de omposition des commissions qui décident?

À partir d'une observation apparemment sans implications cliniques - les neurones immatures ont des concentrations élevées en chlore intracellulaire et cela s'accroît encore après des crises d'épilepsies -, on en est arrivé à proposer un diurétique, qui évacue le chlore intracellulaire, pour traiter des épilepsies du nourrisson ${ }^{1}$. On comprend mieux pourquoi le phénobarbital, qui reste la molécule de première intention, aggrave pourtant souvent les crises. Encore plus étonnant peut-être, nous avons pu montrer [4] que, lors de l'accouchement, l'hormone ocytocine qui le déclenche génère aussi une baisse des niveaux de chlore intracellulaire, entraînant une véritable anesthésie préparatoire du futur bébé et une plus grande résistance aux épisodes anoxiques qui interviennent parfois. Ce qui a aussi d'importantes implications. La détermination d'un bon mécanisme aboutit presque toujours à des applications... après un délai raisonnable.

Comme dirait Isaac Asimov «la connaissance pose des questions auxquelles l'ignorance ne répond jamais ». $\diamond$

Research administration, gene therapy and bench to bed research translation

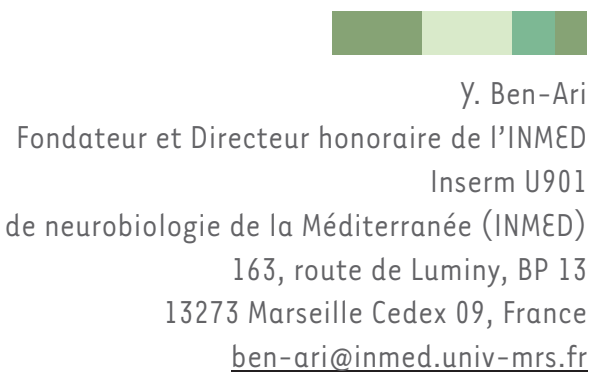

CONFLIT D'INTÉRÊTS

L'auteur déclare n'avoir aucun conflit d'intérêts concernant les données publiées dans cet article.

\section{RÉFÉRENCES}

1. Ben-Ari Y. Neuro-archaeology: pre-symptomatic architecture and signature of neurological disorders. Trends Neurosci $2008 ; 31: 626-36$.

2. Manent JB, Wang $Y$, Chang $Y$, et al. Dcx reexpression reduces subcortical band heterotopia and seizure threshold in an animal model of neuronal migration disorder. Nat Med 2009; 15 : 84-90.

3. Ben-Ari Y, Gaiarsa JL, Tyzio R, Khazipov R. GABA: a pioneer transmitter that excites immature neurons and generates primitive oscillations. Physiol Rev 2007 ; 87 : 1215-84.

4. Tyzio $R$, Cossart R, Khalilov I, et al. Maternal oxytocin triggers a transient inhibitory switch in GABA signaling in the fetal brain during delivery. Science $2006 ; 314: 1788-92$.

${ }^{1}$ Projet européen FP7-HEALTH-2009-4.2-1: Adapting off-patent medicines to the specific needs of paediatric populations, collaborative project (NEMO).

TIRÉS À PART

Y. Ben-Ari

\section{Yehezkel Ben Ari, premier chercheur français lauréat du Prix Européen de l’Épileptologie 2010}

La Commission européenne de la Ligue Internationale Contre l’épilepsie (International League Against Epilepsy - ILAE) distinguera pour la première fois un chercheur fondamentaliste français, le professeur Yehezkel Ben Ari en lui remettant le Prix européen de l'Épileptologie 2010 à Rhodes le 27 juin 2010 en ouverture du 9e congrès européen en Épileptologie. 


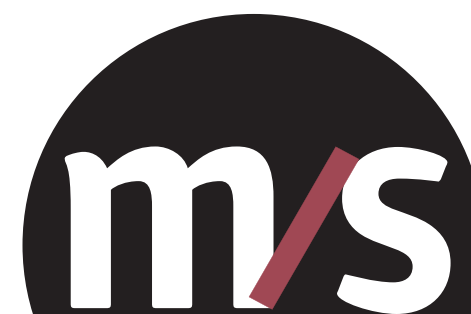

MEDECINE SCIENCES

Chaque mois, avec les articles de référence de $\mathrm{M} / \mathrm{S}$

\section{Chaque jour, sur www.medecinesciences.org}

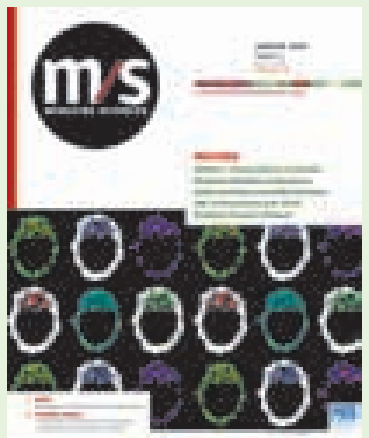

Médecine/Sciences est indexé dans

\section{PubMed/Medline}

Current Contents, série Life Sciences EMBASE/Excerpta Medica

PASCAL

CABS

BIOSIS

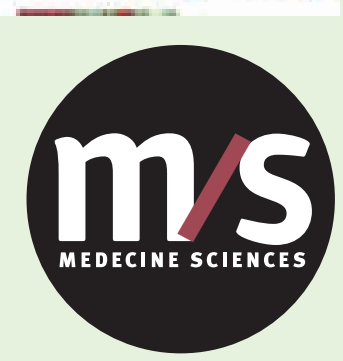

Tarifs d'abonnement M/S - 2010

Mensuel - 10 numéros/an

Abonnez-vous

à Médecine/Sciences
> Des articles rédigés par des médecins et des chercheurs reconnus sur la scène internationale qui posent avec rigueur les bases des débats scientifiques.

> Des synthèses, éditoriaux, dossiers techniques et analyses toujours replacés dans leur contexte pour que l'information soit la plus exacte, intelligible et objective.

> La dimension humaine privilégiée, avec l'analyse des retombées diagnostiques, thérapeutiques, la prévention et l'éthique liées aux nouvelles avancées.

> Un panorama clair et concis de l'actualité scientifique: des nouvelles, des brèves, des données chiffrées, des repères et perspectives pour qu'aucun fait significatif ne vous échappe.
Je souhaite m'abonner à $M / S$ :

Nom:

Adresse :

Code postal

Pays :

$\varepsilon$-mail-obligatoire

Je choisis l'abonnement:

\begin{tabular}{cc}
\multicolumn{2}{c}{ Particuliers } \\
Papier & Électronique \\
+ & seul \\
Électronique &
\end{tabular}

France

$\square 185 €$

$U \varepsilon+$ autres

$\square 245 €$

$\square 125 €$

$\square 125 €$

* Joindre un justificatif
Prénom :

Ville

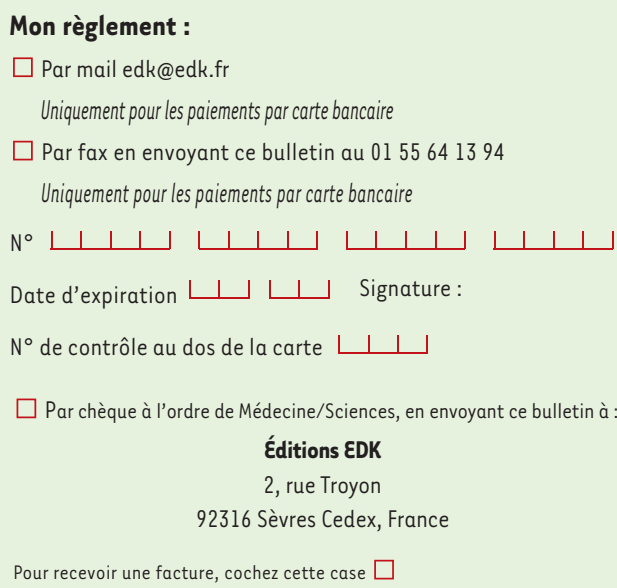




\title{
Ateliers de formation 2010
}

Renseignements et inscriptions :

Ateliers de formation Inserm

101, rue de Tolbiac

75654 Paris Cedex 13

Tél. : 33 (0)1 44236204 - Fax : 33 (0)1 44236293

ateliers@inserm.fr

www.rh.inserm.fr

\section{mantust Int Inserm \\ Institut national \\ Se la sante et de la recherche midicale}

WWW. rh.insermifr

\section{Atelier de formation $\mathrm{n}^{\circ} 206$}

\section{Dynamique des microtubules et migration cellulaire : interactions moléculaires, conséquences fonctionnelles et perspectives thérapeutiques en cancérologie}

\author{
Organisateurs : Stéphane Honoré (Inserm U91 1, CRO2); Diane Braguer (Inserm U91 1, CRO2)
}

\section{Phase I - Le point sur...}

15-17 septembre 2010 - Saint-Raphaël

Objectifs - L'atelier a pour objectif de faire le point sur la méthodologie et les connaissances des mécanismes moléculaires et cellulaires impliquant le cytosquelette microtubulaire dans la migration cellulaire. Nous nous intéresserons particulièrement aux propriétés dynamiques des microtubules et à leur régulation par les complexes protéiques associés à l'extrémité + des microtubules (+TIPs). Nous aborderons notamment la biologie structurale et cellulaire des + TIPs : interactions moléculaires +TIPs/microtubules et +TIPs/+TIPs, les modifications post-traductionnelles de la tubuline et des +TIPs et leurs conséquences fonctionnelles sur la dynamique des microtubules et la coordination des cytosquelettes.

L'atelier fera également le point sur les approches pharmacologiques ciblant les complexes protéiques à l'extrémité + des microtubules et présentera les stratégies en développement pour le criblage de nouveaux composés spécifiques des +TIPs pour des applications en cancérologie.

Public - Chercheurs, cliniciens, pharmacologues, post-doctorants, doctorants et ingénieurs du milieu académique et du secteur pharmaceutique industriel dont le domaine d'étude porte sur le cytosquelette et la migration cellulaire ou l'identification de nouvelles cibles thérapeutiques anti-migratoires.

Les conférences seront données en anglais.

Programme • - La migration cellulaire dans le processus cancéreux.

- Dynamique des microtubules, coordination des cytosquelettes dans la migration cellulaire.

- Interactions microtubules - +TIPs et +TIPs -+TIPs, conséquences fonctionnelles.

- Approches pharmacologiques : de l'existant aux stratégies de criblage pour des anti-+TIPs

\section{Phase II • Maîtrise technique 27-29 octobre 2010 - Paris}

Programme - - Imagerie et analyse de la dynamique des microtubules et du ciblage des sites d'adhérence sur cellules vivantes (vidéomicroscopie à fluorescence, TIRF).

- Imagerie et dynamique des interactions MT/MAPs (microscopie confocale FRET, FRAP).

- Imagerie et analyse du transport intracellulaire microtubule-dépendent (vidéomicroscopie à fluorescence).

Sélection • 9 participants sélectionnés parmi les participants de la phase I.

Avec la participation de • Anna Akhmanova (Rotterdam, The Netherlands), Annie Andrieux (Grenoble, France), Ali Badache (Marseille, France), Denis Chrétien (Rennes, France), Sandrine Etienne- Manneville (Paris, France), Elaine Fuchs (New-York, USA), Niels Galjart (Rotterdam, The Netherlands), Greg Gundersen (New-York, USA), Stéphane Honoré (Marseille, France), Irina Kaverina (Nashville, USA), Laurence Lafanachère (Grenoble, France), Xavier Morelli (Marseille, France), Véronique Proux (Paris, France), Michel Steinmetz (Villigen, Switzerland).

Date limite d'inscription : 15 juillet 2010 